\title{
ON A SERIES OF INVOLUTORIAL CREMONA TRANSFORMATIONS OF SPACE DEFINED BY A PENCIL OF RULED SURFACES*
}

\author{
BY \\ VIRGIL SNYDER
}

1. Introduction. The transformations here discussed are of interest on account of the peculiar manner in which the fundamental elements enter; these singularities have not been mentioned in the existing literature.

Consider a pencil of surfaces $\left|F_{n}\right|$ having a rational curve $r$ to multiplicity $n-2$ as part of its base curve. Let the points $M$ of $r$ and the surfaces of the pencil $|F|$ be in $(1, k)$ correspondence. A point $P$ fixes a surface $F(p)$ of the pencil and this in turn a point $M$ on $r$. The line $P M$ meets $F(p)$, in one residual point $P^{\prime}$. The relation between $P, P^{\prime}$. is an involutorial birational transformation of space. The case in which the surfaces $F$ are of order $n$, and the base curve an $n-2$ fold line and a single residual curve, have been considered by Carroll. $\uparrow$ The case in which the curve $r$ is of order $>1$ and the residual curve simple, including the (ruled) quartics through a double cubic curve $r$, has been discussed by Black. $\ddagger$

The present paper discusses the possible cases in which every surface of the pencil is ruled. With the exception of the pencil of quartics through a double cubic curve and of the cubics with a common double directrix, the surfaces of the pencil are of order $2 n+m$; the base curve consists of a line $p$ to multiplicity $2(n-1)+m$ and a double curve $r$ of order $n$ meeting the line in n-1 points. Apart from the multiple directrix line and the double curve, the residual base of the pencil consists entirely of generators; each surface of the pencil accounts for $m$ parasitic lines.

The new transformations include a number of well known types, but also many new ones in which $m, n, k$ may each take any positive integral value.

2. Equations. The rational curve $r$ defined parametrically by

$$
x_{1}=f \lambda, x_{2}=f \mu, x_{3}=g, x=h,
$$

wherein $f, g, h$ are binary forms in $\lambda, \mu$ of degree $n-1, n, n$ respectively, meets the line $p: x_{1}=0, x_{2}=0$ in $n-1$ points. Let a point $M \equiv\left(0,0, z_{3}, z_{4}\right)$ on $p$, and a point $(\lambda, \mu)$ on $r$ be in $(2, m)$ correspondence defined by $z_{3}^{2} u+z_{3} z_{4} v+z_{4}^{2} w=0$,

* Presented to the Society, December 29, 1932; received by the editors, October 10, 1932.

$\dagger$ American Journal of Mathematics, vol. 54 (1932), pp. 707-717.

‡ These Transactions, vol. 34 (1932), pp. 795-810. 
wherein $u, v, w$ are binary forms of degree $m$ in $\lambda, \mu$. Lines joining corresponding points generate a ruled surface. Since $x_{1} \mu=x_{2} \lambda$, if we write

$$
f\left(x_{1}, x_{2}\right) x_{3}-g\left(x_{1}, x_{2}\right)=f x_{3}-g \equiv \xi \text {, and } f x_{4}-h \equiv \eta,
$$

its equation has the form

$$
F \equiv \xi^{2} u+\xi \eta v+\eta^{2} w=0 .
$$

The surface is of order $2 n+m$; it contains $r$ to multiplicity 2 and $p$ to multiplicity $2(n-1)+m$. From every point of $p$ issue $m$ generators apart from $p$, and the line $p$ counts for $2(n-1)$ generators, two for each point of intersection of $r$ and $p$.

Let $F^{\prime}=0$ be of the same kind, in which $u, v, w$ are replaced by $u^{\prime}, v^{\prime}, w^{\prime}$ of the same degree $m$, and $f, g, h$ be the same as before. Let surfaces of the pencil

$$
l F^{\prime}-l^{\prime} F=0
$$

and points $M$ of $p$ be in $(k, 1)$ correspondence defined by $z_{3} \phi_{4}\left(l, l^{\prime}\right)-z_{4} \phi_{3}(l$, $\left.l^{\prime}\right)=0$, each $\phi_{i}$ being a fixed binary form of degree $k$.

A point $(y) \equiv\left(y_{1}, y_{2}, y_{3}, y_{4}\right)$ in space uniquely fixes the surface of the pencil (2) passing through it: $l=F(y), l^{\prime}=F^{\prime}(y), z_{3}=\phi_{3}\left(F(y), F^{\prime}(y)\right), z_{4}=\phi_{4}(F(y)$, $\left.F^{\prime}(y)\right)$. A point $(x)$ on the line joining $(y)$ to $M$ has coordinates of the form

$$
\rho x_{1}=\tau y_{1}, \rho x_{2}=\tau y_{2}, \rho x_{3}=\sigma \phi_{3}+\tau y_{3}, \rho x_{4}=\sigma \phi_{4}+\tau y_{4} .
$$

For the point $\left(y^{\prime}\right)$ in which the line meets $F_{y}=0$ again,

$$
\begin{aligned}
& \tau=\left(\xi z_{4}-\eta z_{3}\right) f\left[\xi\left\{z_{8}\left(u^{\prime} v\right)+z_{4}\left(u^{\prime} w\right)\right\}+\eta\left\{z_{3}\left(u^{\prime} w\right)+z_{4}\left(w v^{\prime}\right)\right\}\right], \\
& \sigma=-\left(\xi z_{4}-\eta z_{3}\right)\left[\xi^{2}\left(u^{\prime} v\right)+2 \xi \eta\left(u^{\prime} w\right)+\eta^{2}\left(w v^{\prime}\right)\right],
\end{aligned}
$$

wherein $\left(u^{\prime} v\right) \equiv u^{\prime} v-u v^{\prime}$, etc.

The relation between the points $(y)$ and $\left(y^{\prime}\right)$ is an involutorial Cremona transformation $I$ of space. The factor $\xi z_{4}-\eta z_{3}$ divides out of the transformation. When $z_{3}, z_{4}$ are fixed, $\xi_{z_{4}}-\eta z_{3}=0$ represents a cone containing $r$ and having $M$ on $p$ for vertex. It meets each surface of the pencil (2) belonging to $M$ in the generators passing through $M$, and in the base lines $p$ and $r$ of the pencil.

We may now write, after removing the factor,

$$
\begin{aligned}
& \tau=f\left[z_{3}\left\{\xi\left(u^{\prime} v\right)+\eta\left(u^{\prime} w\right)\right\}+z_{4}\left\{\xi\left(u^{\prime} w\right)+\eta\left(v^{\prime} w\right)\right\}\right], \\
& \sigma=-\left[\xi\left\{\xi\left(u^{\prime} v\right)+\eta\left(u^{\prime} w\right)\right\}+\eta\left\{\xi\left(u^{\prime} w\right)+\eta\left(v^{\prime} w\right)\right\}\right] .
\end{aligned}
$$

Under $I$ every surface of the pencil is transformed into itself. The invariant points are the points of contact of tangents from $M$ to the $k$ surfaces belonging to $M$. But since all the surfaces of the pencil (1) are ruled, if any point is fixed, 
the entire generator passing through the point is fixed. These lines generate the surface $\sigma=0$. The surface $\tau=0$ is the image of the line $p$. It consists of two parts, $f=0$, images of the $n-1$ points of $r$ on $p$, and of a ruled surface, image of the other points of $p$. The generators of each surface of (1) passing through its associated point $M$ are fundamental lines of the second kind. When $(y)$ is chosen on any such line, the point $\left(y^{\prime}\right)$ is not defined, but is the whole line passing through the point (y). As $M$ describes $r$ these lines describe a ruled surface $R$ defined by

$$
R: \xi \phi_{4}-\eta \phi_{3}=0,
$$

which plays the most important part in the transformation.

3. Transformation of the pencil of planes $(p)$. Every plane through $p$ is transformed into itself. In each such plane the involution is of order $2 k+2$; it is of the non-perspective Jonquières type, having the isolated point on $r$ as fundamental point of order $2 k+1$. The plane meets each surface of (1) in $p$ and in two lines which are interchanged by $I$. Among them are two lines which are invariant point by point; the locus of these lines is the surface $\sigma=0$. The class is $k$, as is also evident from the definition of the space transformation. The image of the point $L$ on $r$ consists of $2 k+1$ lines, all belonging to $\tau=0$. The images of the lines of the plane are curves of order $2 k+2$ having $2 k+1$ common tangents at the fundamental point $L$. There are also $2 k+1$ simple fundamental points of the plane transformation on $p$. The lines joining these to $L$ on $r$ are all generators of the surface $R$. Of the $2(k+1)(n-1)$ $+(k+2) m$ tangent planes through $p$ which belong to a general surface of the web of conjugates of the planes of space, $2(k+1)(n-1)+k m$ are fixed for every surface of the web; of these $2(k+1)(n-1)$ are the planes of $f=0$ each counted to multiplicity $2(k+1)$, and the $k m$ other ones are tangent planes of $R$, defined by $y_{3} \phi_{4}-y_{4} \phi_{3}=0$. The $2 m$ variable tangent planes of the conjugate of the plane $(a x)=0$ are defined by

$$
x_{3}\left\{a_{3}\left(u^{\prime} v\right)+a_{4}\left(u^{\prime} w\right)\right\}+x_{4}\left\{a_{3}\left(u^{\prime} w\right)+a_{4}\left(v^{\prime} w\right)\right\}=0 .
$$

Moreover, all the surfaces of the web also touch each other along every one of the $2 k+1$ sheets through $r$. These are defined by $y_{3} z_{4}-y_{4} z_{3}=0$. There is simple contact along $\tau$ and $r$, including all the sheets of each, and $f$ counted $2(k+1)$ times.

When a generator in any plane $\pi$ through $p$ passes through $M$, it is parasitic, that is, a fundamental line of the second kind. The point $M$ is then the conjugate of the other generator in $\pi$. Thus, to each point $M$ of $p$ correspond $m k$ lines, fundamental of the first kind, and the line $p$ is itself multiply parasitic for every point on it. 
4. Residual base elements. The base of the pencil (1) consists of $r$ to multiplicity 2 , of $p$ to multiplicity $2(n-1)+m$, and of $4(n-1)+4 m$ generators. Of these, $4(n-1)$ consist of tangency along $p$ in the planes of $f=0$ and $4 m$ are generators not coincident with $p$. Let $g_{i}$ be one of these latter base generators, and $\pi_{i}$ the plane $p, g_{i}$. Corresponding to every point $M$ on $\dot{p}$ are $k$ residual generators in $\pi_{i}$ associated with $M$, all belonging to a pencil of lines with vertex on $r$. As $M$ describes $p$, these lines generate $\pi_{i}$ in such manner that the complete image of $g_{i}$ is $\pi_{i}$ counted $k$ times.

There are $k+1$ positions of $M$ for which the residual generator on some surface of the pencil (1) passes through it; these are all parasitic lines and are base lines of the web of surfaces conjugate to the planes of space. The composite surface $\tau=0$ consists of the planes $f=0$, images of the $n-1$ points of $r$ on $p$, and of a ruled surface of order $(2 k+1) n+(k+2) m$, having $p$ to multiplicity $(2 k+1) n+(k+2) m-(k+1)$ and $r$ to multiplicity $2 k+1$. The locus $\sigma=0$ of invariant points is a ruled surface of order $2(n+m)$, has $p$ to multiplicity $2(n-1)+2 m$, and $r$ to multiplicity 2 . Since all the fundamental elements are included in $\tau=0, \sigma=0$, the complete configuration can now be accounted for.

5. Table of characteristics. The images of planes and of fundamental elements can now be expressed by the following table:

$$
\begin{aligned}
& s_{1} \sim s_{2(k+1) n+(k+2) m}: p^{2(k+1)(n-1)+(k+2) m} r^{2 k+1} 4 m g_{i} 4 k m g^{\prime} ;
\end{aligned}
$$

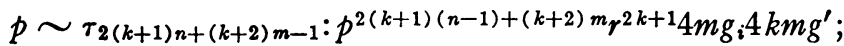

$$
\begin{aligned}
& r \sim R_{(2 k+1) n+k m}: p^{(2 k+1)(n-1)+k m} r^{2 k+1} ; \\
& g_{i} \sim \pi_{i} p g_{i} k g^{\prime} \text {; } \\
& \sigma \equiv \sigma_{2(n+m)}: p^{2(n-1)+2 m} r^{2} 4 m g_{i} 4 k m g^{\prime} ; \\
& J \equiv \tau^{2} R^{2}\left[\left(v u^{\prime}\right)\left(w v^{\prime}\right)-\left(w v^{\prime}\right)^{2}\right] f^{2} .
\end{aligned}
$$

All the surfaces of the web have the same tangent planes along all the $2 k+1$ sheets through $r$. These are the tangent planes of the ruled surface $R$ of fundamental lines of the second kind. The line $p$ illustrates Montesano's theorem for exceptional fundamental lines of the second kind.*

Thus, the complete intersection of any two surfaces of the web consists of the curve conjugate of a line, of the basis elements $p, r$ each to the multiplicity indicated, simple contact along each sheet of $\tau=0$ along $p$, of $R$ along $r$, of $4 m(k+1)$ lines and of each sheet of $f$ counted $2(k+1)$ times.

* D. Montesano, Sulla teoria generale delle corrispondenze birazionali dello spazio, Rendiconti della Accademia dei Lincei, (5), vol. 27 (1918), pp. 396-400 and pp. 438-441; and (5), vol. 302 (1921), pp. $447-451$. 
6. Types not in the preceding category. The preceding list includes all possible types for arbitrary values of $n$ and $m$, but for particular values others may appear.

A pencil of quadrics and an arbitrary line $p$, not a basis line, lead at once to a series of transformations which include one discussed by Montesano.*

The line $p$ may be replaced by any rational curve. The congruence of bisecants of the base $C_{4}$ is left invariant.

The next case is that of a pencil of cubic ruled surfaces having a common double directrix. The residual is then a rational quintic $r_{5}$ meeting the double directrix in four points. The point $M$ is now on $r_{5}$. The residual section of a plane through $d$ and $M$ consists of a generator through $M$ on each of the $k$ surfaces associated with $M$. Each of these lines is parasitic. Every point $P$ of $d$ is invariant except for the plane containing a generator through $P$.

Let $d$ be $x_{3}=0, x_{4}=0$. Then $F_{3} \equiv x_{3}^{2} u+x_{3} x_{4} v+x_{4}^{2} w=0, u=0$ etc. being planes, $F_{3}^{\prime} \equiv x_{3}^{2} u^{\prime}+\cdots=0$.

Let $\mu x_{4}-\lambda x_{3}=0$. Then the parametric equations of $r_{5}$ are

$$
\rho x_{1}=r(\lambda, \mu), x_{2}=s(\lambda, \mu), x_{3}=\lambda f(\lambda, \mu), x_{4}=\mu f(\lambda, \mu),
$$

in which $r$ and $s$ are quintics and $f$ a quartic binary form. Since $f$ is a factor of $\tau$, the image of $d$ includes the $4 k$ surfaces of the pencil associated with the points in which $r_{5}$ meets $d$. The image of the line $d$ is the surface $R \equiv x_{3} \phi_{4}\left(F, F^{\prime}\right)$ $-x_{4} \phi_{3}\left(F, F^{\prime}\right)=0$, of order $3 k+1$ containing $d$ to multiplicity $2 k+1$, and $\tau$ to multiplicity $k$. Every generator is parasitic, hence $R$ also appears as a factor in the transformation. Given a point $P$ on $r_{5}$. The image of $P$ on $F(M)$ is the residual point $P^{\prime}$ in which the line $P M$ meets $F(M)$. As $M$ describes $r_{5}$, this line describes a rational quartic cone, and the locus of $P^{\prime}$ is a curve of order $4 k+N$ having $P$ to multiplicity $N$. The tangent plane to $F(M)$ at $P$ meets $r_{5}$ twice at $P$ and in three other points $K$. Conversely, given $K$, then the line $K P$ and the tangent $t$ to $r_{5}$ at $P$ uniquely fix a tangent plane to $F$, hence the point $M$. The $(1,3 k)$ correspondence between $M$ and $K$ on a rational carrier has $3 k+1$ points of coincidence, hence $P$ is of multiplicity $3 k+1$, and the curve is of order $7 k+1$. In addition, any point $M$ on $r_{5}$ is transformed into the conics through it in the tangent plane to each $F(M)$ at $M$, residual to the generator $g_{M}$. This makes the complete curve of order $9 k+1$. Hence $r$ appears to multiplicity $9 k+1$ on the surfaces conjugate to the planes of space in the involution. This can also be seen directly from the equations of the transformation: Consider a general point $P$ on $d$. It remains invariant on

\footnotetext{
* Su una classe di trasformazioni razionali ed involutorie dello spazio di genere arbitrario $n$ e di grado $2 n+1$, Giornale di Matematiche di Battaglini, vol. 31 (1893), pp. 36-50.
} 
every $F(M)$ of the pencil except when $P M$ is the generator $g_{M}$ on $F(M)$. This happens only for the four positions of $M$ on $d$. The surface $R$ meets any $F$ of the pencil in $d$ counted $4 k+2$ times, in $r$ counted $k$ times, and in a single generator. Let $D$ be one of the points $(d, r)$. Any plane through $d$ meets $F_{(M)}$ in $d^{2}$ and a generator $g$. The image of the generator is $D$. Hence $F_{(M)}$ is the image not of the line $d$ but of the point $D$ on $d$.

$$
\begin{aligned}
s_{1} & \sim s_{27 k+4}: d^{18 k+1} r^{9 k+1} 4 D^{18 k+1} \\
d & \sim R_{3 k+1}: d^{2 k+1} r^{k} 4 D^{2 k+1} \\
r & \sim \tau_{27 k+3}: d^{18 k+1} r^{9 k+1} 4 D^{18 k+1} \\
D & \sim k F_{3}: d^{2 k} r^{k} 4 D^{2 k}
\end{aligned}
$$

The contacts along $d$ and $r$ are as in the general case.

Various particular cases may arise, when $r_{5}$ is composite, consisting of one or more generators and a residual curve. When the generator is taken as a projector curve, the result is included in the preceding category; when the residual curve is the locus of $M$, the image of each generator is a surface of order 6 , found as in the general case. The order of the transformation is lowered by unity for each base generator, since the plane $d, g$ will divide out.

A third particular case is that in which the pencil of quartics have a common double cubic curve. This has been fully treated by Black.*

The space cubic may be replaced by a line and a conic meeting it in one point, or by three lines, one of which meets each of the other two, which are skew. The common transversal is a double generator. When either double directrix is used as projector, this is included in the general case, whether the basal double generator exists or not. When the double generator is used as projector, it is included among those treated by Carroll, the residual basis line now consisting of four simple generators, all skew to the double one. But this case offers differences that warrant a more detailed treatment, since no other generators meet $g$, hence there is no surface of parasitic lines apart from the planes $d, g$ and $d^{\prime}, g$.

Let $d \equiv x_{1}=0, x_{2}=0, d^{\prime} \equiv x_{2}=0, x_{4}=0$ be the two double directrices, and $g \equiv x_{1}=0, x_{2}=0$ the double generator. The equation of the quartic has the form $F_{4} \equiv x_{1}^{2} u+x_{1} x_{2} x_{3} v+a x_{2}^{2} x_{3}^{2}=0$, wherein $u$ is a binary quadratic form in $x_{2}, x_{4}, v$ is linear in $x_{2}, x_{4}$, and $a$ is constant.

Let $u^{\prime}, v^{\prime}, a^{\prime}$ define $F_{4}^{\prime}$ having the same double elements. The pencil $l F^{\prime}-l^{\prime} F=0$ is then associated with the point $M \equiv\left(0,0, z_{3}, z_{4}\right)$ of $g$ by the relation $z_{3} \phi_{4}\left(l, l^{\prime}\right)-z_{4} \phi_{3}\left(l, l^{\prime}\right)=0$, wherein as before $\phi_{i}$ is a binary form in $l$,

* Loc. cit. 
$l^{\prime}$ of degree $k$. The residual base of the pencil consists of four generators $g_{i}$, which meet $d$ and $d^{\prime}$ but do not meet $g$. The pencil contains two composite surfaces, one consisting of the plane $x_{1}=0$ and a ruled cubic containing $d^{\prime}$ as double directrix, and the other of $x_{2}=0$ and a ruled cubic containing $d$ as double directrix. These planes both divide out, each reducing the order of the transformation by one.

The conjugate of the double generator $g$ is generated by the residual conics to surfaces of $|F|$ at $M$. It is a surface of order $8 k+4$, has $d, d^{\prime}$ and $g$ each to multiplicity $4 k+2$, and the simple basic generators $g_{i}$ each to multiplicity $2 k+1$. This surface is ruled.

The surface $\sigma=0$ of invariant points is of order $4 k+5$, has $d, d^{\prime}, g$ each to multiplicity $2 k+1$, and the lines $g_{i}$ each to multiplicity $k+1$.

The image of $g_{i}$ in the plane $M g_{i}$ consists of $k$ nodal cubic curves. As $M$ describes $g$, these curves generate a surface of order $4 k+1$, containing $d$, $d^{\prime}, g$ each to multiplicity $2 k, g_{i}$ to multiplicity $k+1$, and the other basic generators to multiplicity $k$. The images of $d, d^{\prime}$ are the planes $(d, g),\left(d^{\prime}, g\right)$ respectively. The transformation is now completely defined.

CoRNeLl University, ITHACA, N. Y. 